\title{
Upward spirals of the heart: Autonomic flexibility, as indexed by vagal tone, reciprocally and prospectively predicts positive emotions and social connectedness
}

\author{
Bethany E. Kok ${ }^{\star}$ and Barbara L. Fredrickson \\ Department of Psychology, University of North Carolina at Chapel Hill, Chapel Hill, NC United \\ States
}

\begin{abstract}
Vagal tone $(V T)$, an index of autonomic flexibility, is linked to social and psychological wellbeing. We posit that the association between $V T$ and well-being reflects an "upward spiral" in which autonomic flexibility, represented by $V T$, facilitates capitalizing on social and emotional opportunities and the resulting opportunistic gains, in turn, lead to higher VT. Communitydwelling adults were asked to monitor and report their positive emotions and the degree to which they felt socially connected each day for 9 weeks. VT was measured at the beginning and end of the 9-week period. Adults who possessed higher initial levels of $V T$ increased in connectedness and positive emotions more rapidly than others. Furthermore, increases in connectedness and positive emotions predicted increases in $V T$, independent of initial $V T$ level. This evidence is consistent with an "upward spiral" relationship of reciprocal causality, in which VT and psychosocial well-being reciprocally and prospectively predict one another.
\end{abstract}

\section{Keywords}

Rsa; Vagal tone; Emotions; Social support; Self-regulation

\begin{abstract}
Research has linked vagal tone (VT) to numerous indices of psychological well-being, including trait positive emotionality (Oveis et al., 2009), prosocial behavior (Eisenberg et al., 1995), sympathy (Fabes et al., 1993) and decreased maladaptive coping (El-Sheikh et al., 2001). Individuals higher in $V T$ appear to be cheerful and kind and deal well with stress, and these tendencies manifest themselves from childhood1 onward (Porges et al., 1994). What is it that makes individuals high in VT better off than their low-VT counterparts?

Inspired by Fredrickson's $(1998,2001)$ broaden-and-build theory of positive emotions, we posit that the association between $V T$ and well-being reflects a reciprocal causality, an "upward spiral" in which $V T$ facilitates capitalizing on social and emotional opportunities and the resulting opportunistic gains, in turn, lead to higher VT. In such a spiral, the consequences of small, subtle, even fleeting behaviors and emotions accumulate and compound over time, eventually building durable personal resources that significantly improve a person's well-being. In other words, we conceptualize $V T$ as a durable personal resource that moderates the degree to which people experience positive emotions in daily
\end{abstract}

\footnotetext{
(C) 2010 Elsevier B.V. All rights reserved

*Corresponding author. Tel.: +1 919667 4752. bethanyk@unc.edu (B.E. Kok)..

${ }^{1}$ High vagal tone typically manifests in infants as greater behavioral reactivity to their environments, which can be interpreted as "difficultness." Greater reactivity, however, is believed to prepare the infant for greater social success, as reactive or "difficult" infants are apt to receive more social attention from caregivers than their less reactive peers (Porges et al., 1994).
} 
life, and that, over time, experienced positivity bolsters and builds a variety of enduring personal resources, including (but not limited to) VT. Similar upward spiral patterns have been observed for positive emotions and mental health (Fredrickson et al., 2008), positive emotions and coping (Fredrickson and Joiner, 2002; Burns et al., 2008) and positive emotions and trust (Burns et al., 2008), but to date research on upward spirals has not involved physiological factors.

Vagal tone is a promising upward spiral component because it is linked to autonomic flexibility, the capacity of the parasympathetic nervous system (PNS) to adapt to changes in circumstance by modifying arousal, respiration, heart rate and attention (Porges, 1995; Friedman and Thayer, 1998). Individuals high in $V T$ adapt well across a number of different domains. They demonstrate superior performance on numerous indices of cognitive flexibility, including working memory (Hansen et al., 2003), directed attention (Suess et al., 1994) and inhibition of a dominant response (Johnsen et al., 2003; Mezzacappa et al., 1999). They show fewer negative responses to environmental stressors (El-Sheikh et al., 2001), show greater self-regulatory capacity (Segerstrom and Nes, 2007) and are better able to regulate negative facial expressions (Demaree et al., 2004, 2006; Kettunen et al., 2000).

Swift adaptability can influence behavior in subtle yet significant ways. For example, egoresilience is an index of trait-level moment-by-moment adaptability and openness to current circumstances (Block and Kremen, 1996). In two articles, Waugh et al. show that such resilience predicts speed of affective recovery after an anticipated threat fails to emerge. While anticipating threat, all participants experienced increases in negative affect. However, when the threat did not appear, individuals high in resilience were faster to return to baseline in both self-reported emotion (Waugh et al., 2008a) and anterior insula activity, an area associated with anticipatory anxiety (Waugh et al., 2008b). Through their exquisite attunement to the nuances of their current circumstances, resilient individuals are open to opportunities even in the midst of negativity.

Openness to opportunities can lead to greater positivity and social connectedness. In a study of social goals and well-being, approach social goals and motives in college students positively predicted life satisfaction and positive attitudes toward social relationships 8 weeks later, as well as decreased loneliness relative to start of study (Gable, 2006). These effects were mediated by increases in the number of positive life events experienced, suggesting that approach social goals influenced behavior in ways that resulted in positive life outcomes. Similarly, changes in resilience over the course of a month predicted change in life satisfaction (Cohn et al., 2009). Resilient individuals also experience more positive emotions after negative events, though they do not differ in the amount of negative emotions experienced (Fredrickson et al., 2003; Tugade et al., 2004). In all of the studies cited above, initial levels of life satisfaction or positive emotions were controlled for, suggesting that openness helps individuals to get more out of day-to-day opportunities independent of how well or how poorly they were doing at the start of the study.

Just as openness and flexibility predict positivity and social connection, tonic positive emotions and social connectedness predict $V T$. Over the course of an 8-month study of college freshmen, baseline positive emotionality at start as well as positive mood measured 1 month and 6-8 months later were positively associated with VT (Oveis et al., 2009). Individuals with supportive friends show higher $V T$ levels than individuals with ambivalent friends (Holt-Lunstad et al., 2007). In a male-only sample, both trait secure attachment and secure attachment within one's current relationships predicted high VT (Diamond and Hicks, 2005). Porges' polyvagal theory suggests that these behaviors are linked to vagal tone via the social engagement system, a feedback loop between visceromotor vagal efferent pathways regulating the heart and somamotor visceral efferent pathways to brain structures regulating 
musculature in the face and ears associated with emotional expressivity and other-focus (Porges, 1995, 2007).

Both social connectedness and positive emotions, then, are associated with higher $V T$. In addition, $V T$ is a marker of autonomic flexibility and adaptability, characteristics that lead to increases in social connectedness and positive emotions. Assuming that flexibility helps people capitalize on socioemotional opportunities as they arise, we hypothesize that the flexibility indexed by $V T$ over time leads to increased social connectedness and positive emotions. Social connectedness and positive emotions, in turn, bring about incremental increases in $V T$, laying the groundwork for upward spirals toward greater flexibility and well-being.

To test this upward spiral hypothesis, community-dwelling adults were asked to monitor and report their positive emotions and the degree to which they felt socially connected each day for 9 weeks as part of a larger experimental study.2 Measures of $V T$ were taken at the beginning and end of the study. We divided our overarching hypothesis into two testable components: though all participants would gain by capitalizing on the opportunity to reflect (Burton and King, 2007), (1) individuals with higher initial VT are hypothesized to show greater positive change in positive emotions and social connectedness over the course of the study, and (2) increases in positive emotions and social connectedness are hypothesized to predict increases in end-of-study $V T$. In combination, these elements would provide evidence to support our hypothesized psychophysiological upward spiral toward improved well-being.

\section{Method}

Seventy-three adult participants ( $90.4 \%$ white, $40 \%$ male, mean age $=37.3$, range $21-68)$ were recruited from the university community through fliers and email invitation. After giving consent, participants provided a 2-min3 free-respiration vagal tone baseline while alone in a small, quiet room. $V T$ was measured via respiratory sinus arrthymia (RSA), a noninvasive measure of cardiac vagal control characterized by increases in heart rate with inspiration and decreases in heart rate with expiration (Berntson et al., 1997). Continuous recordings were made of heart rate and respiration measures at a sampling rate of $1000 \mathrm{~Hz}$. From these recordings, second-by-second averages were computed. Disposable snap electrodes were placed in a bipolar configuration on lateral sides of the chest on the lowermost ribs to measure the participant's echocardiogram (ECG). Respiration was collected with pneumatic bellows, placed around the participant's chest. All data were inspected offline and corrected for artifacts. RSA was calculated off-line based on changes in heart rate associated with respiration using a modified Grossman peak-to-valley method (Grossman, 1983) with interbeat intervals prorated to an equal sampling interval of $125 \mathrm{~ms}$. Data were excluded if neither a peak or a valley could be identified during a sampling interval. Respiration was statistically controlled for in analyses by using the residual of RSA values when predicted by respiration. 4

\footnotetext{
${ }^{2}$ The data are drawn from a field experiment that investigated the psychological effects of learning to meditate, akin to the experiment reported by Fredrickson et al. (2008). Participants were randomly assigned to attend a 7-week meditation workshop or to serve in a monitoring, waitlist control. All completed a number of measures beyond those reported here. Experimental condition was controlled for in all models.

${ }^{3}$ Before beginning psychophysiological data collection, participants spent several minutes in the cubicle at rest with psychophysiological sensors attached in order to habituate them to their environment and establish a similar physiological state of relaxation for all participants. To confirm stability of measurement over the two minute period, RSA values for the first and second minute were correlated. During the first lab session, $r=.85, p<.0001$. During the second lab session, $r=.91, p<.0001$.

${ }^{4}$ Fourteen participants were missing RSA data at either time 1 or time 2, five participants' RSA scores were discarded due to high ECG impedances and RSA data from one participant was excluded due to an anomalous RSA reading over 5 standard deviations from the mean.
} 
Each day for the next 63 days, participants listed the three social interactions in which they spent the most time that day. They then rated these three interactions in aggregate using 2 items adapted from Russell's UCLA Loneliness scale (1996): "During the social interactions, I felt "in tune" with the person/s around me" and "During the social interactions, I felt close to the person/s," using a 7-point scale $(1=$ not at all true, $7=$ very true). The items were averaged to create a mean social connectedness score (mean daily $\alpha$ $=.94, S D=.03$ ). Participants also gave daily emotion reports using the modified Differential Emotions Scale (mDES; Fredrickson et al., 2003). Participants rated their strongest experiences of 20 emotions in the past day on a 5-point scale $(0=$ not at all to $4=$ extremely). Participants' mean positive emotion score was composed of amusement, awe, gratitude, hope, inspiration, interest, joy, love, pride and serenity (mean daily $\alpha=.94, S D=$. 01). Participants' mean negative emotion score was composed of anger, boredom, contempt, disgust, embarrassment, fear, guilt, hate, sadness and shame (mean daily $\alpha=.85, S D=.07$ ). After 9 weeks, participants provided another two-minute RSA baseline under the same laboratory conditions.

\section{Results}

\section{Preliminary analyses}

The data were first analyzed by computing the per-week mean, standard deviation and number of reports for social connectedness, positive emotions and negative emotions. As shown in Table 1, participants began the study slightly above the scale midpoint on selfrated social connectedness and slightly below the scale midpoint on positive and negative emotions, indicating a minimal likelihood of ceiling effects in the subsequent analyses. We also examined change in the variability in participants' responses over time. Decreasing variability in reported emotions or connectedness over time might suggest that participants had modified their reports in response to perceived demand effects in the study. As shown in Table 1, weekly standard deviations remained relatively stable over time, evidence against the presence of a time-dependent response bias.

\section{Hypothesis 1: VT predicts change in social connectedness and positive (but not negative) emotions over time}

Hierarchical linear growth models with a random slope for time and random intercept were used to track participants' within-and-between-person change over time in the three target variables.

Results are presented in Table 2. As anticipated, after controlling for the effects of gender and experimental condition, participants increased significantly in social connectedness and positive emotions over the course of the study, and decreased significantly in negative emotions. Unexpectedly, $V T$ was a direct predictor of negative emotions, with participants higher in $V T$ showing a higher level of negative emotions throughout the study. In addition, consistent with our hypothesis, participants' $V T$ upon entering the study interacted with time in study to predict a greater increase in social connectedness and positive (but not negative) emotions for participants initially high in $V T$.

\section{Hypothesis 2: Change over time in social connectedness and positive emotions predicts end-of-study VT}

To achieve a per-person change over time score, individual-person growth models were fitted for daily social connectedness and positive emotions and the slopes for each were recorded.5 We then regressed participants' end-of-study $V T$ on their change over time for social connectedness and positive emotions separately, controlling for initial $V T$ and initial 
level of social connectedness or positive emotions. Gender and condition did not significantly predict end-of-study $V T$ and were excluded from the models reported below.

As hypothesized, after controlling for initial $V T$, participants' rate of change in social connectedness over time $(\beta=.93$, one-tailed $p=.02)$ and positive emotions over time ( $\beta=$ 1.01 , one-tailed $p=.04$ ) significantly predicted end-of-study $V T$, while start-of-study social connectedness ( $\beta=-.00$, one-tailed $p=.27)$ and positive emotions $(\beta=.00$, one-tailed $p=$. $45)$ did not. Neither rate of change nor initial level of negative emotions were significant predictors of end-of-study $V T$.

The upward-spiral relations among initial $V T$, end of study $V T$ and change in social connectedness and positive emotions are shown in Fig. 1.

\section{Discussion}

We hypothesized and found that autonomic flexibility, as indexed by vagal tone, predicts gains in positive emotions and social connectedness, which in turn result in greater autonomic flexibility. Specifically, we found that, when given the opportunity to reflect on their experiences each day, adults who possessed higher levels of $V T$ increased in social connectedness and positive emotions more rapidly than others. Furthermore, we found that degree of change in social connectedness and positive emotions predicted change in $V T$, independent of initial $V T$ level, as assessed on study entry.

This evidence is consistent with the hypothesized "upward spiral" relationship of reciprocal causality, in which $V T$ and psychosocial well-being reciprocally and prospectively predict one another. It is also consistent with our assumption that autonomic flexibility, indexed by $V T$, promotes well-being and further gains in flexibility by helping people capitalize on social and emotional opportunities as they arise in daily life. If this upward spiral and skill in capitalizing are established early in life, they could help to explain the superior social, cognitive and psychological abilities exhibited by children, adolescents and adults with higher-than-average $V T$.

Unexpectedly, we also found a direct effect of $V T$ on negative emotions. Participants higher in $V T$ at the beginning of the study reported a higher level of negative emotions throughout the 9 weeks. Because $V T$ has not been positively associated with negative emotions in previous research (Oveis et al., 2009; Rottenberg et al., 2007), we speculate that this finding may be due to chance. It is also possible, however, that high-VT participants reported more negative emotions because data were collected over the holiday season, a time high in social stresses that can be exacerbated by emotional closeness to others. The potential vulnerability of high- $V T$ individuals to negative social environments calls for further study.

Our findings illuminate key boundary conditions on Fredrickson's broaden-and-build theory of positive emotions. Specifically, the pattern of results suggest that $V T$ is a gateway personal resource: it moderates the degree to which people experience positive emotions and social connection in daily life, which in turn determines the degree to which they experiences the broaden-and-build benefits of such positive experiences, such as, in this case, increased $V T$. Other personal resources may similarly moderate the positive emotion yield of recurrent activities - like social interaction or other wellness-enhancing behaviors and thus function as gateways to upward spiral processes posited by the broaden-and-build

\footnotetext{
${ }^{5}$ An SEM model was fitted to test hypothesis 1 and hypothesis 2 simultaneously, but there was insufficient information for the models to converge when using daily data. We attribute this to sample size.
} 
theory. For instance, we speculate that high levels of oxytocin or low levels of inflammation might similarly increase both positive emotions as well as their sequelae.

One limitation of this study stemmed from the need to control for an unrelated experimental manipulation in the analyses; while experimental condition did not appear to play a role, this additional predictor necessarily decreased power in our statistical models. In addition, the relatively small sample size limited the scope of our analyses. For instance, we were unable to test both parts of the upward spiral hypothesis simultaneously in one model without collapsing PE and SC scores across weeks, an action that significantly reduces the power of the combined model to detect effects.

This work used intensive longitudinal sampling to explore part of the day-to-day lives of individuals high and low in $V T$, an approach that proved fruitful in elucidating potential micro-level differences in experience that, upon accumulation, may contribute to greater well-being. To achieve this level of detail, however, our area of focus was necessarily narrow and excluded many elements of daily experience. Because $V T$ is linked to adaptability, future studies could investigate responses to daily negative experiences, particularly coping with negative social experiences. Methodologically, peer reports of emotionality and social behavior could be used to corroborate the patterns seen here.

Participants also increased in positive emotions and social connectedness over the course of the study independent of vagal tone, as depicted by the main effects of time in Table 2.

Having not observed main effects of time on self-reported positive emotions in past studies (Fredrickson et al., 2008; Cohn et al., 2009), we speculate that this global trend reflected the initial listing activity, in which participants made a list of their three longest social interactions before evaluating their level of social connectedness each day. Prior research has shown that as little as two minutes of reflective writing can have beneficial effects on health (Burton and King, 2007). Further experimental work is needed to test the psychological benefits of briefly reflecting on daily social interactions.

We have speculated that autonomic flexibility, or adaptability, contributes to well-being by encouraging capitalization on social and emotional opportunities. Future studies that include explicit measures of capitalization could assess this postulated mediating mechanism, and potentially link the physiological measure of vagal tone to a particular pattern of behavioral responses to environmental opportunities.

One important question regarding upward spirals is whether individuals might eventually achieve a "ceiling," the highest possible amount of growth, or whether there is the possibility of "too much" growth. Research on mania certainly suggests that when it comes to positive emotions, there can be too much of a good thing (Gruber et al., 2008). In addition, Fredrickson and Losada's modeling of upward spirals of positivity in team performance suggests that such spirals may become unstable at extremely high ratios of positive to negative emotions (2005). Such a ratio (11.6 positive emotions for every one negative emotion), however, is dependent not only on experiencing many positive emotions but also on avoiding negative emotions. To the extent that some negative emotion is both healthy and unavoidable (Schwartz and Clore, 2003), individuals who experience their emotions genuinely and without suppressing negative feelings are unlikely to reach such a high ratio.

The capacity for adaptability indexed by $V T$ is relevant to a wide range of behavioral domains. We speculate that high VT may help to explain why some individuals are better able to adapt to life changes such as moving to a new city or starting a new exercise routine. Knowing in advance whether a person is likely to need additional assistance and support for positive behavior changes to "take" would be highly beneficial for health care providers, 
human resource managers, counselors and others whose aim is to help individuals capitalize on opportunities to achieve positive psychological and health outcomes. The implication that autonomic flexibility can be enhanced over time is also good news for those individuals who may struggle with adapting to change.

In sum, "upward spirals" in well-being may occur in part through the recruitment of $V T$, an index of flexibility, adaptability and openness to opportunities. By virtue of their ability to make appropriate psychophysiological adjustments "on the fly," individuals high in VT may cut a path to well-being that not only goes through the social world, but also through the heart.

\section{Acknowledgments}

This research was funded with the support of National Institute of Mental Health Grant MH59615 to the second author. Portions of this manuscript were presented as part of the first author's master's thesis, and have been presented at scientific conferences, including Association for Psychological Science and Society of Personality and Social Psychology. We thank Jolynn Pek for statistical guidance and comments on earlier drafts. We also thank James Long for consultation regarding electrophysiological measurement.

\section{References}

Berntson GG, Bigger JJ, Eckberg DL, Grossman P, Kaufmann PG, Malik M, et al. Heart rate variability: origins, methods, and interpretive caveats. Psychophysiology. 1997; 34(6):623-648. [PubMed: 9401419]

Block J, Kremen AM. IQ and ego-resiliency: conceptual and empirical connections and separateness. Journal of Personality and Social Psychology. 1996; 70:349-361. [PubMed: 8636887]

Burns AB, Brown JS, Sachs-Ericsson N, Plant EA, Curtis JT, Fredrickson BL, Joiner TE. Upward spirals of positive emotion and coping: replication, extension, and initial exploration of neurochemical substrates. Personality and Individuals Differences. 2008; 44:360-370.

Burton CM, King LA. The effects of (very) brief writing on health: The two-minute miracle. British Journal of Health Psychology. 2007; 00:1-7.

Cohn MA, Fredrickson BL, Brown SL, Mikels JA, Conway AM. Happiness unpacked: positive emotions increase life satisfaction by building resilience. Emotion. 2009; 9(3):361-368. [PubMed: 19485613]

Demaree HA, Pu J, Robinson JL, Schmeichel BJ, Everhart DE. Predicting facial valence to negative stimuli from resting RSA: not a function of active emotion regulation. Cognition and Emotion. 2006; 20(2):161-176.

Demaree HA, Robinson JL, Everhart DE, Schmeichel BJ. Resting RSA is associated with natural and self-regulated responses to negative emotional stimuli. Brain and Cognition. 2004; 56(1):14-23. [PubMed: 15380871]

Diamond LM, Hicks AM. Attachment style, current relationship security, and negative emotions: the mediating role of physiological regulation. Journal of Social and Personal Relationships. 2005; 22:499-518.

Eisenberg N, Fabes RA, Murphy B, Maszk P, Smith M, Karbon M. The role of emotionality and regulation in children's social functioning: a longitudinal study. Child Development. 1995; 66(5): 1360-1384. [PubMed: 7555221]

El-Sheikh M, Harger JA, Whitson SM. Exposure to interparental conflict and children's adjustment and physical health: the moderating role of vagal tone. Child Development. 2001; 72(6):16171636. [PubMed: 11768136]

Fabes RA, Eisenberg N, Eisenbud L. Behavioral and physiological correlates of children's reactions to others' distress. Developmental Psychology. 1993; 29:655-663.

Fredrickson BL. What good are positive emotions? Review of General Psychology. 1998; 2(3):300319.

Fredrickson B. The role of positive emotions in positive psychology: the broaden-and-build theory of positive emotions. American Psychologist. 2001; 56(3):218-226. [PubMed: 11315248] 
Fredrickson BL, Cohn MA, Coffey KA, Pek J, Finkel SM. Open hearts build lives: positive emotions, induced through loving-kindness meditation, build consequential personal resources. Journal of Personality and Social Psychology. 2008; 95(5):1045-1062. [PubMed: 18954193]

Fredrickson BL, Joiner T. Positive emotions trigger upward spirals toward emotional well-being. Psychological Science. 2002; 13:172-175. [PubMed: 11934003]

Fredrickson BL, Losada MF. Positive affect and the complex dynamics of human flourishing. American Psychologist. 2005; 60(7):678-686. [PubMed: 16221001]

Fredrickson BL, Tugade MM, Waugh CE, Larkin GR. What good are positive emotions in crises? A prospective study of resilience and emotions following the terrorist attacks on the United States on September 11th 2001. Journal of Personality and Social Psychology. 2003; 84(2):365-376. [PubMed: 12585810]

Friedman BH, Thayer JF. Anxiety and autonomic flexibility: a cardiovascular approach. Biological Psychology. 1998; 47(3):243-263. [PubMed: 9564452]

Gable SL. Approach and avoidance social motives and goals. Journal of Personality. 2006; 71:175222. [PubMed: 16451230]

Grossman P. Respiration, stress, and cardiovascular function. Psychophysiology. 1983; 20:284-300. [PubMed: 6408680]

Gruber J, Johnson SL, Oveis C, Keltner D. Risk for mania and positive emotional responding: too much of a good thing? Emotion. 2008; 8(1):23-33. [PubMed: 18266513]

Hansen AL, Johnsen BH, Thayer JF. Vagal influence in the regulation of attention and working memory. International Journal of Psychophysiology. 2003; 48:263-274. [PubMed: 12798986]

Holt-Lunstad J, Uchino BN, Smith TW, Hicks A. On the importance of relationship quality: the impact of ambivalence in friendships on cardiovascular functioning. Annals of Behavioral Medicine. 2007; 33(3):278-290. [PubMed: 17600455]

Johnsen BH, Thayer JF, Laberg JC, Wormnes B, Raadal M, Skaret E, Kvale G, Berg E. Attentional and physiological characteristics of patients with dental anxiety. Journal of Anxiety Disorders. 2003; 17:75-87. [PubMed: 12464290]

Kettunen J, Ravaja N, Näätänen P, Keltikangas-Järvinen L. The relationship of respiratory sinus arrhythmia to the co-activation of autonomic and facial responses during the Rorschach test. Psychophysiology. 2000; 37(2):242-250. [PubMed: 10731774]

Mezzacappa E, Kindlon D, Saul JP, Earls F. Relations of age to cognitive and motivational elements of impulse control in boys with and without externalizing behavior problems. Journal of Abnormal Child Psychology. 1999; 27:473-483. [PubMed: 10821629]

Oveis C, Cohen AB, Gruber J, Shiota MN, Haidt J, Keltner D. Resting respiratory sinus arrhythmia is associated with tonic positive emotionality. Emotion. 2009; 9(2):265-270. [PubMed: 19348538]

Porges SW. Orienting in a defensive world: Mammalian modifications of our evolutionary heritage. A polyvagal theory. Psychophysiology. 1995; 32(4):301-318. [PubMed: 7652107]

Porges SW. The polyvagal perspective. Biological Psychology. 2007; 74(2):116-143. [PubMed: 17049418]

Porges SW, Doussard-Roosevelt JA, Portales AL, Suess PE. Cardiac vagal tone: Stability and relation to difficultness in infants and three-year-olds. Developmental Psychobiology. 1994; 27:289-300. [PubMed: 7926281]

Schwartz N, Clore GL. Mood as Information: 20 Years Later. Psychological Inquiry. 2003; 14(3\&4): 296-303.

Segerstrom SC, Nes LS. Heart rate variability reflects self-regulatory strength, effort, and fatigue. Psychological Science. 2007; 18(3):275-281. [PubMed: 17444926]

Rottenberg J, Clift A, Bolden S, Salomon K. RSA fluctuation in major depressive disorder. Psychophysiology. 2007; 44(3):450-458. [PubMed: 17371497]

Russell DW. The UCLA Loneliness Scale (Version 3): Reliability, validity, and factor structure. Journal of Personality Assessment. 1996; 66:20-40. [PubMed: 8576833]

Suess PE, Porges SW, Plude DJ. Cardiac vagal tone and sustained attention in school-age children. Psychophysiology. 1994; 31:17-22. [PubMed: 8146250] 
Tugade MM, Fredrickson BL, Feldman Barrett L. Psychological resilience and positive emotional granularity: Examining the benefits of positive emotions on coping and health. Journal of Personality. 2004; 72:1161-1190. [PubMed: 15509280]

Waugh CE, Fredrickson BL, Taylor SF. Adapting to life's slings and arrows: Individual differences in resilience when recovering from an anticipated threat. Journal of Research in Personality. 2008a; 42:1031-1046. [PubMed: 19649310]

Waugh CE, Wager TD, Fredrickson BL, Noll DC, Taylor SF. The neural correlates of trait resilience when anticipating and recovering from threat. Social Cognitive and Affective Neuroscience. 2008b; 3(4):322-332. [PubMed: 19015078] 


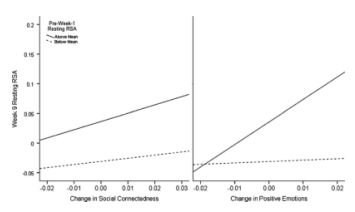

Fig. 1.

Post-test respiration-corrected RSA as predicted by pre-test respiration-corrected RSA together with changes in social connectedness (A) and positive emotions (B). Units of the $X$ axis represent each participant's average change in social connectedness or positive emotions per day. Units of the $Y$ axis represent residualized RSA scores after controlling for respiration. 


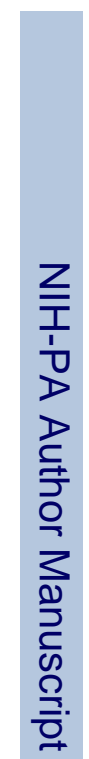

Kok and Fredrickson

Page 11

$\frac{\overline{0}}{\frac{0}{\circ}}$

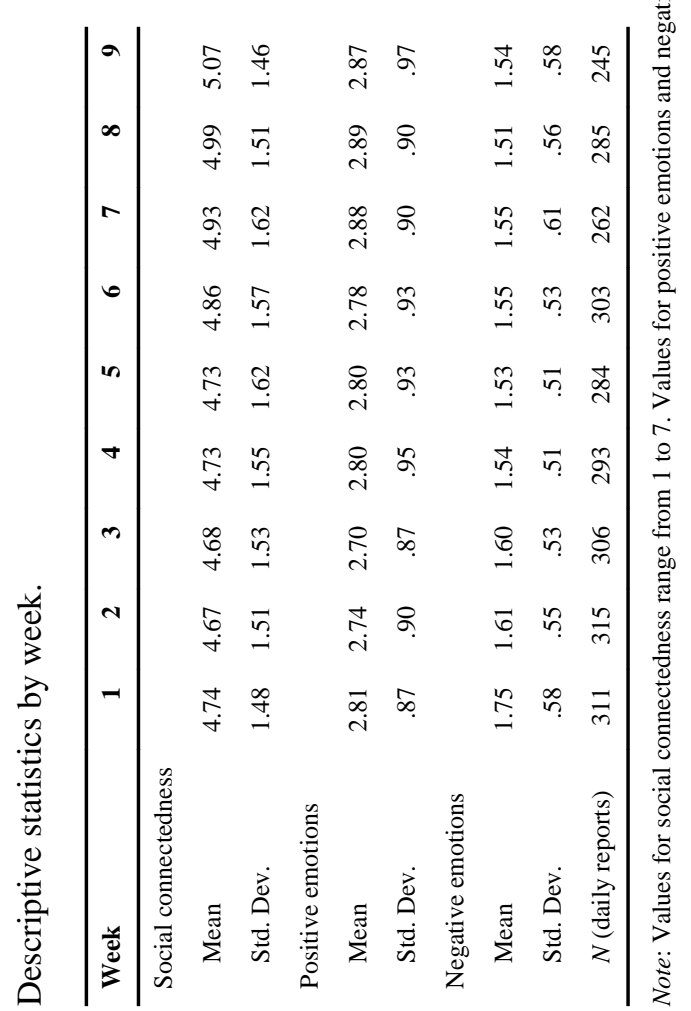

Biol Psychol. Author manuscript; available in PMC 2011 June 24. 


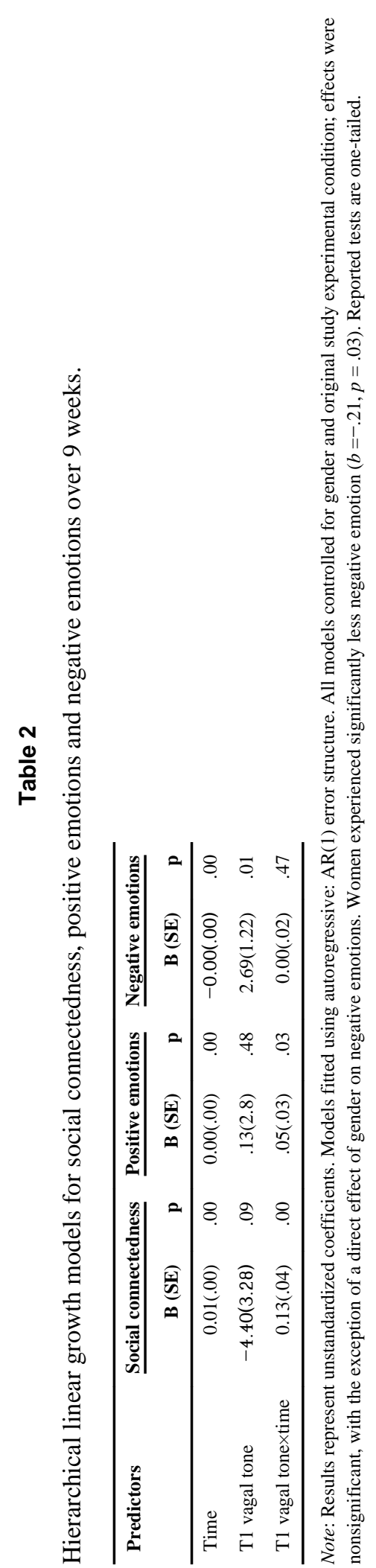

Biol Psychol. Author manuscript; available in PMC 2011 June 24. 